\title{
One-Pot Reactions: Expeditious Synthesis of Diverse Organic Compounds of Current Interests
}

\section{Bimal Banik $\mathbf{K}^{1,2,3,4^{*}}$}

${ }^{1}$ Department of Chemistry and Chemical Biology, Stevens Institute of Technology, Hoboken, New Jersey, USA

${ }^{2}$ The University of Texas MD Anderson Cancer Center, USA

${ }^{3}$ Department of Chemistry, The University of Texas-Pan American, USA

${ }^{4}$ Community Health Systems of South Texas, Edinburg, Texas 78539, USA

\begin{abstract}
Organic compounds are in general synthesized by multiple steps and as a result, isolation and purification of the compounds after each step is necessary. Therefore, synthesis of unknown organic molecules becomes expensive and the whole process becomes time-consuming. To design reactions that can be done in a one-pot method is necessary. In continuation of our studies on antibiotics, anticancer agents and diverse heterocycles, this perspective demonstrates a few one-pot methods for the synthesis of numerous compounds of current interests.
\end{abstract}

Keywords: One pot method; Beta lactam; Michael reaction; PaalKnorr reaction; Hydrogenation; Microwave

\section{Introduction}

Synthesis of organic compounds mostly requires multiple steps, several extractions, purifications and characterizations. To make a process shorter, it is necessary to design methods very carefully. Toward the goal, to develop one-pot synthesis is ideal. In some instances, onepot synthesis may become feasible if the reactants, products, solvents and reagents does not interfere each other during the process.

\section{Discussion}

\section{Synthesis of optically active Schiff bases}

Schiff bases are widely used in organic synthesis because of the presence of a reactive $\mathrm{C}=\mathrm{N}$ functional group in their structures. Particularly, these types of compounds are prepared by reaction of carbonyl compounds and primary amines through a dehydration process. If the amines and carbonyl compounds are available from the market or not rare chemicals their direct reactions in the presence of a mild acid or drying agent can produce Schiff bases easily. D-Glyceraldehyde is an important carbohydrate-derived aldehyde and this compound is used extensively in the synthesis of diverse natural and non-natural products including new -lactams. The usual procedure for the preparation and isolation of D-glyceraldehyde is a complicated process. However, it was found that this aldehyde can be prepared in aqueous solution by reacting 1:2, 5:6-Di-O-isopropyledene $\mathrm{D}$-mannitol with sodium periodate [1]. The reactivity of D-glyceraldehyde is extremely high even in the presence of water. For examples, several primary amines were able to form optically active imines (Schiff bases) with D-glyceraldehyde in water solution in excellent yield. Thus, isolation of D-glyceraldehyde from water solution was not necessary.

\section{Synthesis of racemic $\beta$-lactams}

Synthesis of new $\beta$-lactams is the subject of intense investigation for the past many decades because these types of compounds have a wide range of medicinal activities. Parallel to numerous studies by other groups, we have been conducting exploratory research on $\beta$-lactams for the past 27 years [2,3]. Although there are many methods known for the synthesis of $\beta$-lactams, cycloaddtion of imines with activated acid chloride (equivalent) in the presence of a tertiary base is proved to be most demanding. However, this reaction requires isolation of imines and is conducted in the presence of organic solvents. Therefore, a onepot synthesis of new $\beta$-lactams may add considerable importance on this subject further. Montmorillonite, a solid clay is also a dehydrating agent. Diverse imines were obtained from aromatic amines and aromatic aldehydes in the presence of montmorillonite [4]. No solvents were necessary for this reaction. The progress of the reaction was followed through IR and TLC. The reactivity was dependent on the functional group of amines and carbonyl compounds. One-pot synthesis of $\beta$-lactams without isolating the imines was possible. We envisioned that the clay and other reagents used for the imine formation may not disturb any reactions with the reagents necessary for the next step.3, 4-Disubstituted $\beta$-lactams was produced by the reaction of the in situ generated Schiff base with an acid chloride (for example, acetoxyacetyl chloride, benzyloxyacetyl chloride, crotonyl chloride and phenoxyacetyl chloride) and a tertiary base (for example, triethylamine and N-methylmorpholine) [2]. After the reaction was completed, the mixture was filtered and the solid mass was washed with methylene chloride. The organic part was evaporated and the residue showed the presence of $\beta$-lactams. Interestingly, the diastereoselectivity of the $\beta$-lactams obtained through the one-pot method remained the same with the compound prepared by cycloaddition following step-wise processes.

\section{Reduction of the double bond and N-C4 bond cleavage in $\beta$-lactams}

Unsaturated $\beta$-lactams were hydrogenated to prepare saturated $\beta$-lactams by catalytic reduction method. $\beta$-Lactams that have an aromatic group at the $\mathrm{C}-4$ position of the ring were hydrogenated and cleaved to open chain amides by catalytic transfer hydrogenation method [5]. Ammonium formate in the presence of $10 \% \mathrm{Pd} / \mathrm{C}$ was found to be the best reagent for this purpose. Importantly, this reaction was conducted in a domestic microwave oven within 1-2 minutes. The presence of an aromatic group was required for the success of this method to obtain open chain amides. However, reduction of the olefinic groups was possible by this method regardless of their

*Corresponding author: Bimal Banik K, Community Health Systems of South Texas, Edinburg, Texas 78539, USA, Tel: 19566274094; E-mail: bimalbanik10@gmail.com (or) bimal.banik@chsst.org

Received August 03, 2017; Accepted August 05, 2017; Published August 10, 2017

Citation: Bimal Banik K (2017) One-Pot Reactions: Expeditious Synthesis of Diverse Organic Compounds of Current Interests. Mod Chem appl 5: 229. doi: 10.4172/2329-6798.1000229

Copyright: ( 2017 Bimal Banik K. This is an open-access article distributed under the terms of the Creative Commons Attribution License, which permits unrestricted use, distribution, and reproduction in any medium, provided the original author and source are credited. 
position in the $\beta$-lactam rings. This one-pot method was used for the preparation of diverse alkyl/aryl substituted amides of unique structures. This reaction did not proceed if the aromatic group at C-4 position is sterically hindered. Thus, such $\mathrm{N}-\mathrm{C} 4$ bond cleavage was not possible with multicyclic fused $\beta$-lactams in which an aromatic system was present. From a limited study, chemoselectivity of the reduction of the unsaturated group and $\mathrm{N}-\mathrm{C} 4$ bond cleavage was not identified.

\section{Hydrogenolysis and $\mathrm{N}-\mathrm{C} 4$ bond cleavage in $\beta$-lactams}

In another one-pot reaction, hydrogenolysis of the benzyl ether group along with $\mathrm{N}-\mathrm{C} 4$ bond cleavage in $\beta$-lactams was also performed successfully by catalytic transfer hydrogenation. This reaction produced hydroxy-substituted amides. It was not possible to differentiate the rate of hydrogenolysis of the benzyl ether and $\mathrm{N}-\mathrm{C} 4$ bond cleavage [5]. It appeared both of these reactions take place simultaneously.

\section{Saturation of the double bond and allylic deacetoxylation in $\beta$-lactams}

The one-pot method was extended to $\beta$-lactams bound to a carbohydrate. It was found that catalytic transfer hydrogenation method worked well in the reduction of the alkene group present in a glycoside of a $\beta$-lactam as well as in the deacetoxylation of an allylic acetate group of the carbohydrate in a one pot operation [6]. Furthermore, reduction of the alkene bond, deacetoxylation and $\mathrm{N}-\mathrm{C} 4$ bond cleavage were also observed in specific substrates in a one-pot method. It was not possible to identify the chemoselectivity among these processes.

\section{Deprotection, oxidation and esterification in $\beta$-lactams}

Deprotection of a relatively unstable ketal or sugar group in a $\beta$-lactam with iodine to a diol, oxidation of the diol with ruthenium trichloride/sodium periodate to an acid and esterification of the acid with diazomethane to an ester was conducted following onepot method [7]. The yield of the ester was high. In another one-pot method for the same target molecule, deprotection of a ketal group was achieved with p-tolunesulfonic acid to a diol, oxidation of the diol with lead tetracetate to an aldehyde and further oxidation with chromium trioxide to an acid and esterification of the acid with diazomethane.

\section{Deprotection and rearrangement to lactone in $\beta$-lactams}

Optically active $\beta$-lactam with a ketal group at C-4 position of the ring was deprotected to a diol by iodine-catalyzed reaction or p-toluenesulfonic acid and the diol was converted to a lactone upon treatment with trifluroacetic acid (TFA) following a one-pot method. TFA was able to cleave the ring of the $\beta$-lactam and the resulting amino acid derivative formed a 5-membered lactone by reaction with the secondary hydroxy group present in the diol [7]. No 6-membered lactone was formed.

\section{Hydroxy $\beta$-lactams to amino $\beta$-lactams}

Optically active 3 -hydroxy- $\beta$-lactam was converted to its mesylate with methane sulfonyl chloride in the presence of triethylamine, the mesylate was then transformed to an azide by treatment with lithium azide, and finally the azide was reduced to an amino $\beta$-lactam by hydrogen sulfide in a one-pot method. There was an inversion of configuration of the stereochemistry of the ring system during mesylate displacement reaction by azide [7].

\section{Reduction and rearrangement in $\beta$-lactams}

The aromatic nitro group in $\beta$-lactam was reduced to an amino derivative by zinc and ammonium chloride. This amino compound was isolated. However, reduction of the same nitro group with indium/ ammonium chloride in aqueous ethanol afforded oxazines through a molecular rearrangement in a one-pot method. This reductionrearrangement by indium metal confirmed the coordinating power of the metal with the carbonyl group of the lactam. This type of coordination helped the amino group to attack the carbonyl group due to an increase of its nucleophilic character [8].

\section{Deprotection, synthesis of pyrroles and Michael reaction}

Paal-Knorr method required a primary amine, an active diketone and acidic catalysts for the preparation of $\mathrm{N}$-substituted pyrroles. We demonstrated the synthesis of pyrroles starting from 2,5-dimethoxytetrahydrofuran and primary amine in the presence of acidic catalysts [9]. The furan derivative was degraded to a reactive 1, 4-dialdehyde which was then condensed with amines to form $\mathrm{N}$-substituted pyrroles. Addition of 2, 3-unstatuated aldehydes or ketones to the reaction mixture was successful to form 2-substututed product through Michael reaction in a one-pot method [10].

\section{Michael reaction of indoles and reduction of ketone in indoles}

Bismuth nitrate-catalyzed Michael reaction of indole with unsaturated ketones was investigated [10]. The addition reaction took place at the C-3 position of indole. The keto group present in these products was subsequently reduced to alcohols by sodium borohydride in a one-pot operation. The protection of the keto group as ketal was also performed without separating the Michal adduct.

\section{Polycyclic aromatic hydrocarbons}

Diverse hydrofluorenes were prepared following one-pot reaction. Alkylaton of ketone with a benzyl bromide in the presence of a strong base, cyclization of the ketone with the aromatic group, and aromatization were the steps involved in the whole processes [11]. For example, alkylation of 2-tetralone with 2-napthyl bromide in the presence of sodium hydride was performed. The resulting alkylated ketone was cyclized to the pentacyclic compound through cyclodehydration in the presence of strong sulfuric acid. Prolonged exposure in the presence of strong acid underwent complete aromatization to dibenzofluorene. Similarly other polyaromatic fluorene were prepared in a one-pot reaction. Some of the derivatives prepared from these polyaromatic compounds demonstrated anticancer activity [12]

\section{Microwave-induced methods}

Microwave-induced reactions are widely accepted procedure in science. There are a number of advantages of conducting microwave reactions than reactions by classical methods. A number of methods discussed here were performed in domestic microwave oven with great success [13]. Development of one-pot reactions in microwave oven had enhanced the visibility of these chemical processes. In addition to cut down the costs to produce compounds of interests, these methods are environmentally friendly and rapid.

\section{Conclusion}

One-pot synthesis of organic compounds is undoubtedly very versatile and convenient. However, it is not possible to perform all sequences of reactions following this method. It is obvious that the functional groups present in certain molecules may have great affinity to one of the unwanted reagents for different other reactions or cannot tolerate the reaction conditions. Therefore, to design one-pot method, scientists should be careful to know the function of the intermediate structures, role of the catalysts/reagents, incoming reactants and also on the composition of the whole reactions including the solvent used. 
Citation: Bimal Banik K (2017) One-Pot Reactions: Expeditious Synthesis of Diverse Organic Compounds of Current Interests. Mod Chem appl 5: 229. doi: $10.4172 / 2329-6798.1000229$

Page 3 of 3

Nevertheless, it is also possible to perform 2-4 reactions sequentially in a one-pot method.

\section{Acknowledgements}

Bimal Krishna Banik is grateful to many students and scientists and his mentors who participated in these projects. He is also grateful to $\mathrm{NIH}, \mathrm{NCl}$, Kleberg Foundation, Stevens Institute of Technology, University of Texas MD Anderson Cancer Center, University of Texas-Pan American, University of Texas Health Science Center at San Antonio and Community Health System of South Texas for providing the necessary funding and laboratory space.

\section{References}

1. Banik BK, Manhas MS, Kaluza Z (1992) Microwave-induced Organic Reaction Enhancement Chemistry Convenient Synthesis of Enantiopure Hydroxy- $\beta$ Lactams. Tetrahedron Lett 33: 3603-3606.

2. Banik BK (2010) Heterocyclic Scaffolds I. Top Heterocycl Chem Springer 22: 1-379.

3. Banik I, Becker FF, Banik BK (2003) Stereoselective Synthesis of $\beta$-Lactams with Polyaromaic Imines: Entry to New and Novel Anticancer Agents. J Med Chem 46: 12-15.

4. Ramos K, Banik BK (2011) Microwave-Induced Clay-Mediated Preparation of Imines: One-Pot Synthesis of $\beta$-Lactams. Heterocyclic Letters, pp: 27-30.
5. Banik BK, Barakat KJ, Wagle DR, Manhas MS (1999) Microwave-Assisted Rapid and Simplified Hydrogenation. J Org Chem 64: 5746-5753.

6. Banik BK, Manhas MS, Bose AK (1994) Stereospecific Glycosylation via Ferrier Rearrangement for Optical Resolution. J Org Chem 59: 4714-4716.

7. Bose AK, Banik BK, Mathur C, Wagle DR (2000) Polyhydroxy Amino Acid derivatives via $\beta$-Lactams Using Enantiospecific Approaches and Microwave Techniques. Tetrahedron 56: 5603-5619.

8. Banik BK, Samajdar S, Banik I (2003) Indium-Induced Facile Rearrangement of $\beta$-Lactams to Oxazines. Tetrahedron Lett 44: 1699-1701.

9. Rivera S, Bandyopadhyay D, Banik BK (2009) Facile Synthesis of N-Substituted Pyrroles via Microwave-Induced Bismuth Nitrate-Catalyzed Reaction Under solventless Conditions. Tetrahedron Lett 50: 5445-5448.

10. Srivastava N, Banik BK (2003) Bismuth Nitrate-Catalyzed Versatile Michae Reactions. J Org Chem 68: 2109-2114.

11. Banik BK, Mukhopadhyay C, Becker FF (2001) An Expeditious One-Pot Synthesis of Hydrofluorene Derivatives. Synthetic Communications 31: 2399-2403.

12. Becker FF, Mukhopadhyay C, Hackfeld L (2000) Polycyclic Aromatic Compounds as Anticancer Agents: Synthesis and Biological Evaluation of Dibenzofluorene Derivatives. Bio org Med Chem 8: 2693-2699.

13. Bose AK, Banik BK (1995) Stereocontrol of $\beta$-Lactam Formation Using Microwave Irradiation. Tetrahedron Lett 36: 213-216. 